\title{
Model of creation of productive agrocenosis of Echinacea
}

\author{
Sergey Pospelov*, Viacheslav Zdor, Oleg Mishchenko, Anna Pospelova, and Ninel \\ Kovalenko \\ Poltava State Agrarian Academy, 36003 ul. Skovorody 1/3 Poltava, Ukraine
}

\begin{abstract}
The results of many years of field research on the effect of stocking density of different types of Echinacea spp. on its productivity are discussed. It was found that when the Echinacea purpurea crops were thickened, the collected raw material had a high percentage (more than $50 \%$ ) of stems, which negatively affected its quality. An increase in the density of Echinacea pallida crops has less effect on the formation of generative shoots. Long-term research has revealed patterns that determine the productivity of Echinacea spp. at different planting densities. This made it possible to calculate and recommend for production the density of plants at which the optimal yield of the agrocenosis will be achieved: for Echinacea purpurea - 100-110 thousand/ha, and for Echinacea pallida 120-140 thousand/ha.
\end{abstract}

\section{Introduction}

Representatives of the Echinacea genus (Echinacea Moench) are known in the world primarily as medicinal plants with pronounced immunostimulating properties [1]. This is due to unique phytochemical characteristics, among which the main components are chicoric acid and its derivatives, polysaccharides and alkylamides [1,2]. Due to this, drugs with anti-inflammatory, adaptogenic and immunocorrecting activity are produced from echinacea all over the world $[1,2]$. Today, during a pandemic, these properties of echinacea deserve special attention. It should not be forgotten that echinacea is an excellent honey plant and an ornamental perennial plant $[3,4]$.

Due to the increased demand for raw materials, echinacea has been successfully grown in America and Europe for over a century [2,3]. However, only three species can be seen in culture: Echinacea purpurea (L.) Moench, Echinacea pallida (Nutt.) Nutt. and Echinacea angustifolia DC. Echinacea purpurea is the most studied and used. Although, there are few pharmaceutical varieties in the world, it has mainly a decorative value [3]. Echinacea angustifolia is a raw material for many drugs; but it is harvested more in nature, in places of natural growth such as Canada and North America [4]. In our opinion, Echinacea pallida is the most interesting but underestimated species both in terms of its chemical composition and biological characteristics [2,4-5]. At the Poltava State Agrarian Academy, due to many years of purposeful work, varieties of Echinacea purpurea ('Zirka Mykoly Vavylova') and

\footnotetext{
* Corresponding author: sergii.pospielov@pdaa.edu.ua
} 
Echinacea pallida ('Krasunya of Prairie') were developed, technologies for growing and using Echinacea for the needs of pharmacy, animal husbandry, beekeeping, and ornamental gardening were developed [5].

One of the "narrow" issues of cultivation is the creation of productive plantations [6]. If we consider echinacea mainly as a medicinal plant, then it is impractical to grow it for more than two to three years. Therefore, it is necessary to create optimal accommodations and intensive care during the first year of life in order to get the maximum return in the future [7-12]. It is to these questions that our article is devoted.

\section{Materials and methods}

The research was carried out in the botanical garden of the Poltava National Pedagogical University named after V.G. Korolenko. During 2006-2015, field experiments were carried out, obtained data were processed and interpreted. Investigations on placement schemes (plant density) $45 \times 10,45 \times 20,45 \times 30,70 \times 10,70 \times 20,70 \times 30$ centimeters were laid out for three production cycles in a row by seedling on typical medium-humus chernozems of heavy texture. For this, the seedlings were preliminarily grown in cassettes until they had up to two to four true leaves. Due to this, the plants did not get sick and took root $100 \%$. The productivity of the aboveground mass was assessed during mass flowering, the productivity of the root system was assessed after the end of the growing season. The counts were performed in the third year of the growing season. Correlation analysis and construction of regression equations were performed using MS Excel.

\section{Results and discussion}

The results of the studies showed that the intensity of the formation of generative shoots in Echinacea purpurea depended on the feeding area. Compacted crops resulted in fewer shoots. 3.3-4.0 shoots were formed in the variant where the distance between the plants was $10 \mathrm{~cm}$, and 4.6-7.3 shoots were formed when the indicated interval was increased. There was also a definite tendency towards an increase in the height of the shoots and the size of the inflorescences, and a decrease in the number of inflorescences and leaves with the layouts of $45 \times 10 \mathrm{~cm}$ and $70 \times 10 \mathrm{~cm}$.

The productivity (wet weight) and the structure of the yield of the aboveground mass of Echinacea purpurea depending on the placement schemes are presented in Table 1. With the frequent placement of plants $(45 \times 10 \mathrm{~cm})$, stems comprised the largest percentage in the raw material - $51.4 \%$, which, as you know, reduces its quality. The content of leaves and inflorescences in the raw material was $34.4 \%$ and $14.2 \%$, respectively, and the productivity of one plant was 158.7 grams. The weight of the stems in the variants with the placement of $45 \times 30 \mathrm{~cm}$ and $70 \times 30 \mathrm{~cm}$ was the maximum, but their share in the raw material did not exceed $48.7 \%$. In these variants, there was a significant increase in the mass of leaves of the plants $(68.2-78.5 \mathrm{~g})$ in comparison with the placement schemes of $45 \times 10 \mathrm{~cm}$ and $70 \times 10 \mathrm{~cm}(54.6-55.1 \mathrm{~g})$. The content of leaves in the raw material in all variants was quite stable $-29.1 \%-34.4 \%$. The mass of inflorescences varied significantly according to the placement schemes, its significant increase was noted only on the schemes of $45 \times 30 \mathrm{~cm}$ and $70 \times 20 \mathrm{~cm}$ : 49.2-59.3 g versus 22.5-41.5 grams (when the plants were placed every $10 \mathrm{~cm}$ ). The percentage of inflorescences in the raw material was $14.2 \%-$ $23.6 \%$, depending on the experimental scheme. 
Table 1. Productivity of the aboveground mass of Echinacea purpurea and its structure, (mass/percentage) for different placement schemes (average over three cycles).

\begin{tabular}{|l|c|c|c|c|c|c|}
\hline \multirow{2}{*}{ Indicators } & \multicolumn{5}{|c|}{ Experiment settings (landing patterns, cm) } \\
\cline { 2 - 8 } & $45 \times 10$ & $45 \times 20$ & $45 \times 30$ & $70 \times 10$ & $70 \times 20$ & $70 \times 30$ \\
\hline $\begin{array}{l}\text { 1.Weight of stems, g, } \\
\text { Lsd } 0.05=15.2\end{array}$ & $\underline{81.56}$ & $\frac{96.40}{41.4}$ & $\frac{108.10}{48.7}$ & $\frac{78.97}{45.0}$ & $\frac{85.69}{45.5}$ & $\frac{128.26}{48.2}$ \\
\hline $\begin{array}{l}\text { 2. Weight of leaves, g, } \\
\text { Lsd0.05 = 8.7 }\end{array}$ & $\underline{54.65}$ & $\frac{68.20}{34.4}$ & $\frac{64.54}{29.1}$ & $\frac{55.12}{31.4}$ & $\frac{61.78}{32.8}$ & $\frac{78.50}{\frac{29.5}{2}}$ \\
\hline $\begin{array}{l}\text { 3. Weight of inflorescences, g } \\
\text { Lsd0.05 }=5.2\end{array}$ & $\underline{22.51}$ & $\frac{35.72}{14.2}$ & $\frac{49.19}{22.2}$ & $\frac{41.51}{23.6}$ & $\frac{40.75}{21.7}$ & $\frac{59.34}{22.3}$ \\
\hline $\begin{array}{l}\text { Weight of the aboveground part, } \\
\text { g, }\end{array}$ & $\frac{\mathbf{1 5 8 . 7 2}}{\mathbf{1 0 0}}$ & $\frac{\mathbf{2 0 0 . 3 2}}{\mathbf{1 0 0}}$ & $\frac{\mathbf{2 2 1 . 8 2}}{\mathbf{1 0 0}}$ & $\frac{\mathbf{1 7 5 . 6 3}}{\mathbf{1 0 0}}$ & $\frac{\mathbf{1 8 8 . 2 2}}{\mathbf{1 0 0}}$ & $\frac{\mathbf{2 6 6 . 1 0}}{\mathbf{1 0 0}}$ \\
\hline
\end{tabular}

Echinacea pallida has fewer generative shoots compared to Echinacea purpurea: in experiments, their number was 2.67-5.67 depending on the layout. The mass of generative shoots varied from $69.3 \mathrm{~g}$ to $132.9 \mathrm{~g}$. When placing plants with a row spacing of $45 \mathrm{~cm}$, this indicator in the $45 \times 10 \mathrm{~cm}$ variant was significantly lower $(69.3 \mathrm{~g}$ or $49.4 \%)$ in comparison with other variants (119.1-132.9 g). When placed with a row spacing of $70 \mathrm{~cm}$, the opposite relationship was observed (Table 2). In this case, the number of stems in the raw material was minimal in the variant of $70 \times 30 \mathrm{~cm}(30.9 \%)$ compared with the experimental variants of $70 \times 10$ and $70 \times 20 \mathrm{~cm}(40.1-49.0 \%)$.

Table 2. Productivity of the aboveground mass of Echinacea pallida and its structure, (weight/percentage) for different placement schemes (average for three cycles).

\begin{tabular}{|l|c|c|c|c|c|c|}
\hline \multirow{2}{*}{ Indicators } & \multicolumn{5}{|c|}{ Experiment settings (landing patterns, cm) } \\
\cline { 2 - 7 } & $45 \times 10$ & $45 \times 20$ & $45 \times 30$ & $70 \times 10$ & $70 \times 20$ & $70 \times 30$ \\
\hline $\begin{array}{l}\text { 1. Weight of stems, g, } \\
\text { Lsd } 0.05\end{array}=32.5$ & $\frac{69.29}{49.4}$ & $\frac{132.92}{53.2}$ & $\frac{119.10}{50.0}$ & $\frac{123.26}{49.0}$ & $\frac{94.90}{40.1}$ & $\frac{74,80}{30.9}$ \\
\hline $\begin{array}{l}\text { 2. Weight of leaves, g, } \\
\text { Lsd } 0.05=12.6\end{array}$ & $\frac{36.50}{26.0}$ & $\frac{60.25}{24.1}$ & $\frac{59.80}{25.1}$ & $\frac{56.40}{22.4}$ & $\frac{60.50}{25.6}$ & $\frac{65.70}{27.2}$ \\
\hline $\begin{array}{l}\text { 3. Weight of inflorescences, g, } \\
\text { Lsd0.05 }=28.7\end{array}$ & $\frac{34.36}{24.6}$ & $\frac{56.80}{22.7}$ & $\frac{59.30}{24.9}$ & $\frac{72.03}{28.6}$ & $\frac{81.30}{34.3}$ & $\frac{101.20}{41.9}$ \\
\hline $\begin{array}{l}\text { Weight of the aboveground part, } \\
\text { g }\end{array}$ & $\frac{\mathbf{1 4 0 . 1 5}}{\mathbf{1 0 0}}$ & $\frac{\mathbf{2 4 9 . 9 7}}{\mathbf{1 0 0}}$ & $\frac{\mathbf{2 3 8 . 2 0}}{\mathbf{1 0 0}}$ & $\frac{\mathbf{2 5 1 . 6 9}}{\mathbf{1 0 0}}$ & $\frac{\mathbf{2 3 6 . 7 0}}{\mathbf{1 0 0}}$ & $\frac{\mathbf{2 4 1 . 7 0}}{\mathbf{1 0 0}}$ \\
\hline
\end{tabular}

When placing plants with row spacing of $70 \mathrm{~cm}$, the mass of leaves varied less in comparison with the row spacing of 45 centimeters (56.4-65.7 grams versus 36.5-60.3 grams). The percentage of leaves in the raw material was quite stable and amounted to $22.4-27.2 \%$. The row spacing influenced the weight of the inflorescence the most. When the plants were planted according to the $45 \times 10 \mathrm{~cm}$ scheme, the inflorescences were small and the weight of each plant was $34.4 \mathrm{~g}$ (24.6\% of the total weight). With the schemes of 45 x $20 \mathrm{~cm}$ and $45 \times 30 \mathrm{~cm}$, the quantity of inflorescences significantly increased to 56.8-59.3 $\mathrm{g}$, which corresponded to $22.7-24.9 \%$ of the total plant. Placement with row spacing of 70 
$\mathrm{cm}$ led to a significant increase in the mass of inflorescences on the plant to 72.0-101.2 grams. The highest rates were noted on the $70 \times 30 \mathrm{~cm}$ variant, where the part of flowering baskets in the raw material was $41.9 \%$.

In general, while analyzing the data on the mass of the entire plant of Echinacea pallida, it can be noted that the area of nutrition did not significantly affect its productivity. In experiments, the smallest indicator of 140.2 grams was observed with a planting pattern of $45 \times 10 \mathrm{~cm}$. In other variants, the weight of one plant was 236.7-251.7 grams. It should be noted that in the $70 \times 30 \mathrm{~cm}$ variant, the raw material was of the highest quality in terms of the yield structure, which is explained by the low content of the stems and high content of the inflorescences.

Figure 1 shows the results of studies of the effects of placement schemes on the productivity of the echinacea root system. They indicate a higher productivity of Echinacea purpurea compared to Echinacea pallida. This is especially noticeable in variants with a row spacing of 45 centimeters. Wider row spacings eliminate the difference, mainly due to the increase in the productivity of rhizomes of Echinacea pallida. When the plants were placed according to the scheme of $70 \times 30 \mathrm{~cm}$, the productivity of the aboveground part and the root system of both types of echinacea was practically at the same level, which indicated the significant role of the spatial placement of the culture.

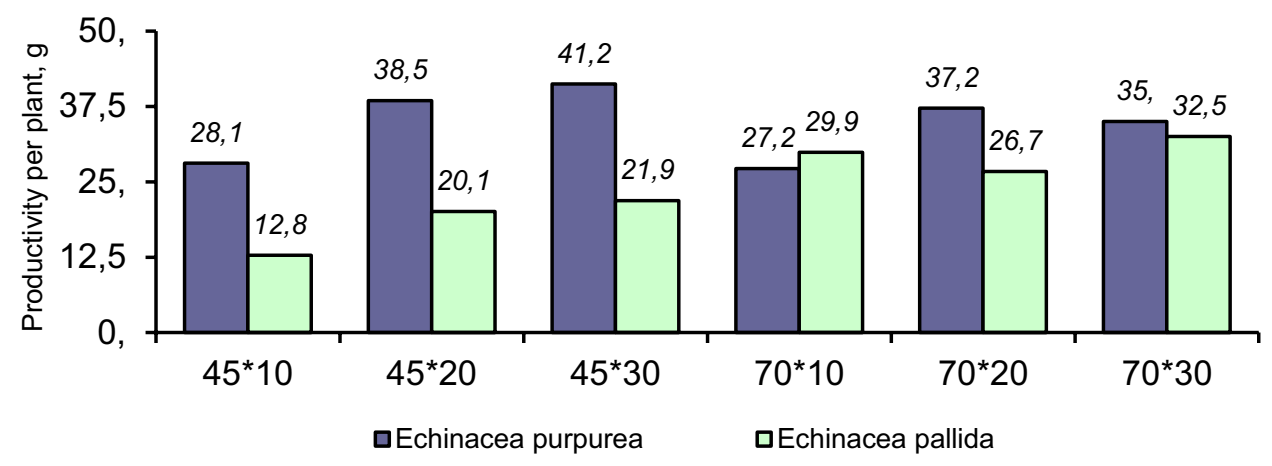

For Echinacea purpurea $-\operatorname{Lsd}_{0.05}=6.2$; for Echinacea pallida $-\operatorname{Lsd}_{0.05}=3.8$

Fig. 1. Productivity of echinacea's rhizomes with roots depending on placement schemes (average for three cycles).

The correlation analysis was performed to analyze the above data, which served as the basis for building the correlation pleiads (Fig. 2, 3) that reflect the relationship between the most significant indicators. For Echinacea purpurea, the mass of the aerial part had the most significant correlations with the mass of stems $(r=0.879)$, the number of inflorescences $(r=0.735)$, and the mass of the root system $(r=0.815)$. The mass of the root system also had high correlation with the mass of inflorescences, width and length of the leaf blade.

Echinacea pallida had much lower performance indicators. The weight of the aerial part had significant correlations with the weight of stems $(r=0.929)$, weight of inflorescences $(r$ $=0.859)$, weight of leaves $(r=0.787)$ and leaf area $(r=0.787)$. The mass of the root system had no significant correlations with other parameters of the plant. 


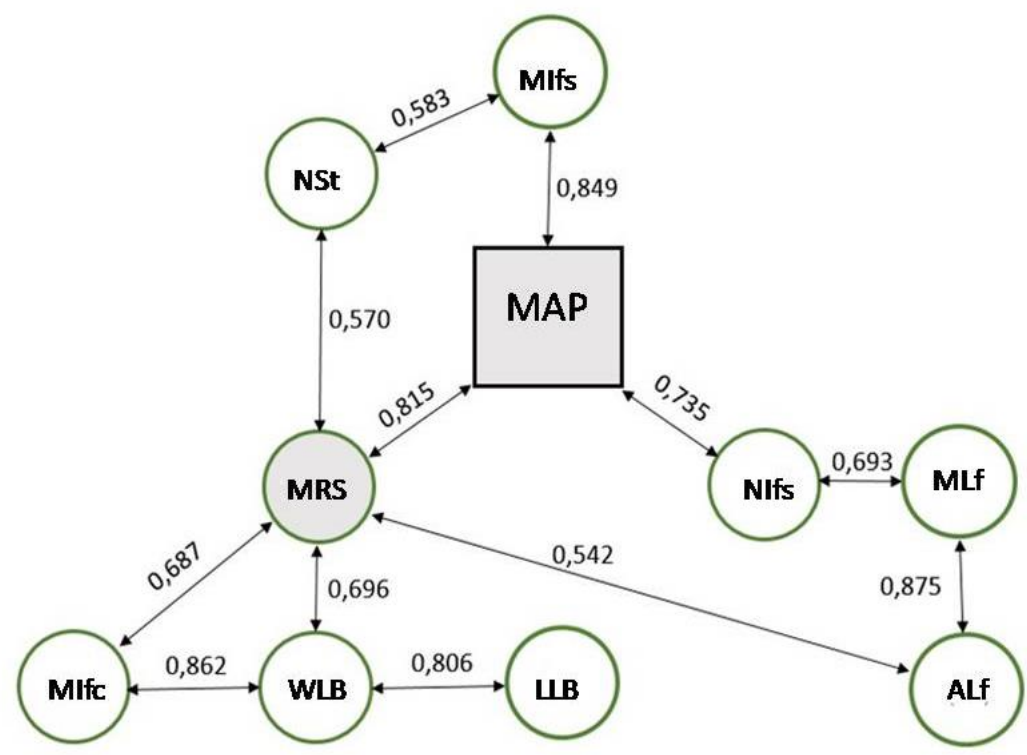

Notes: MAP - mass of the aboveground part; MRS - mass of the root system; ALf - area of the leaf; MSt - mass of stems; MLf - mass of leaves; MIfs - mass of inflorescences; NSt - number of stems; NIfc - the number of inflorescences; LLB - length of the leaf blade; WLB - width of a leaf blade.

Fig. 2. Correlation pleiad of factors determining the productivity of Echinacea purpurea.

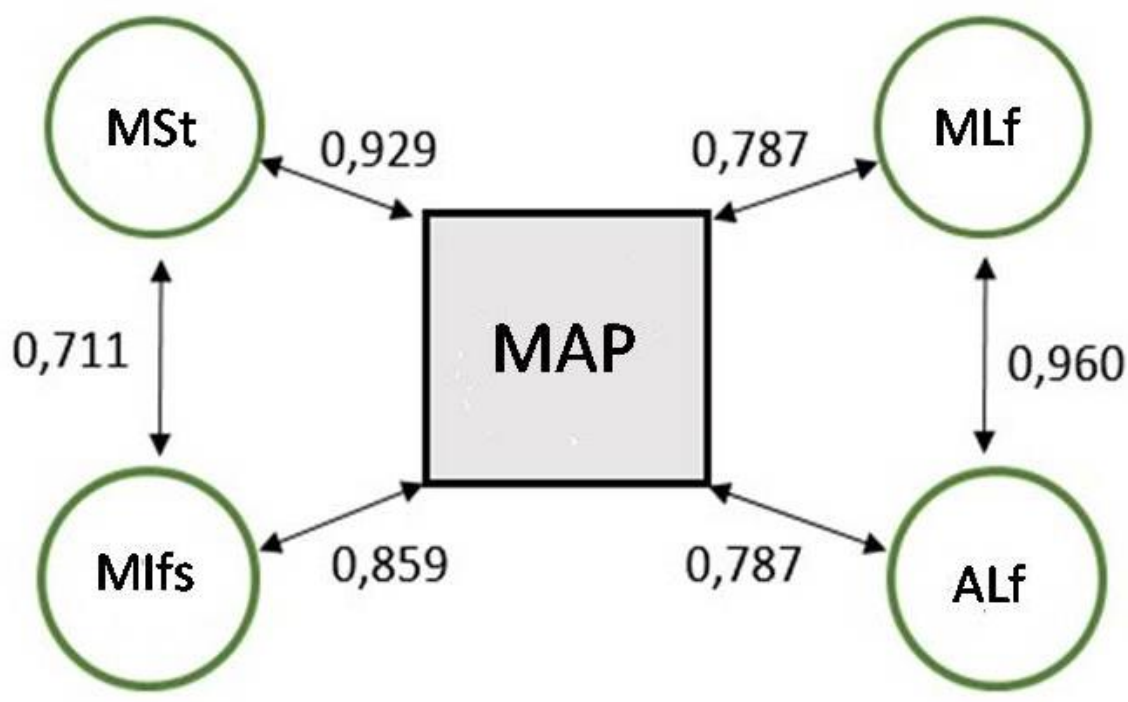

Notes : MAP - mass of the aboveground part; ALf - area of the lea; MSt - mass of stems; MLf - mass of leaves; MIfc - mass of inflorescences.

Fig. 3. Correlation pleiad of factors determining the productivity of Echinacea pallida.

Based on the calculations of correlations between the productivity of the plants and the parameters of their placement, depending on the experimental schemes, a model of the 
optimal agrocenosis of Echinacea was developed. For this, graphic images of the most accurate regression equations were used when approximating experimental data (Fig. 4, 5).
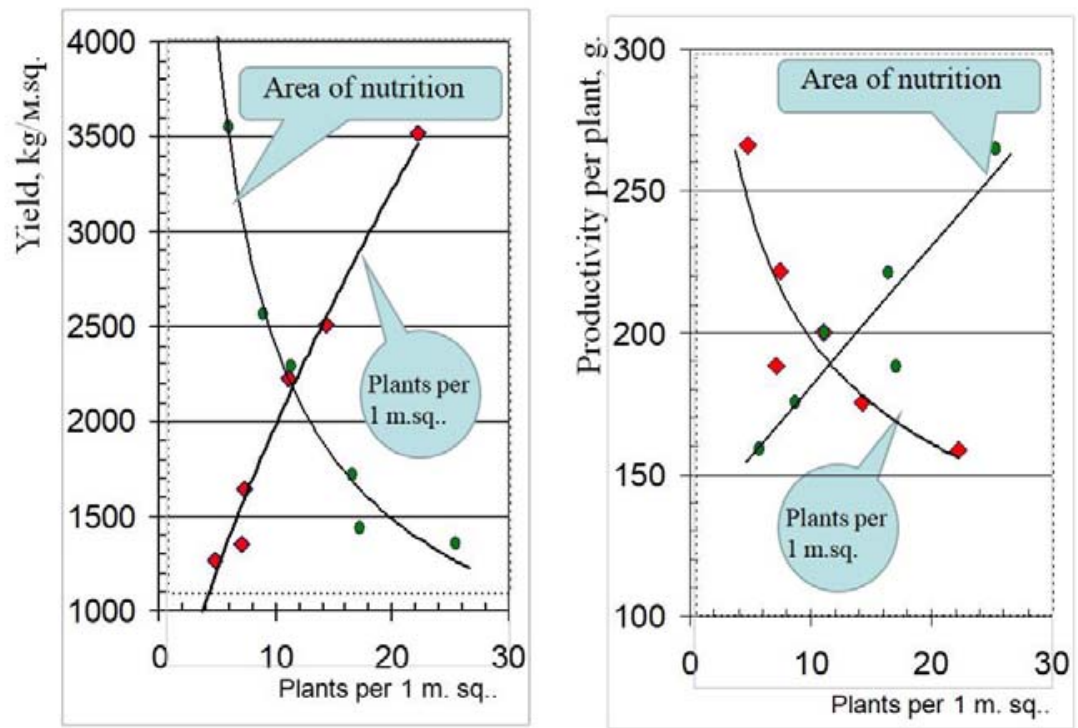

A - aboveground part
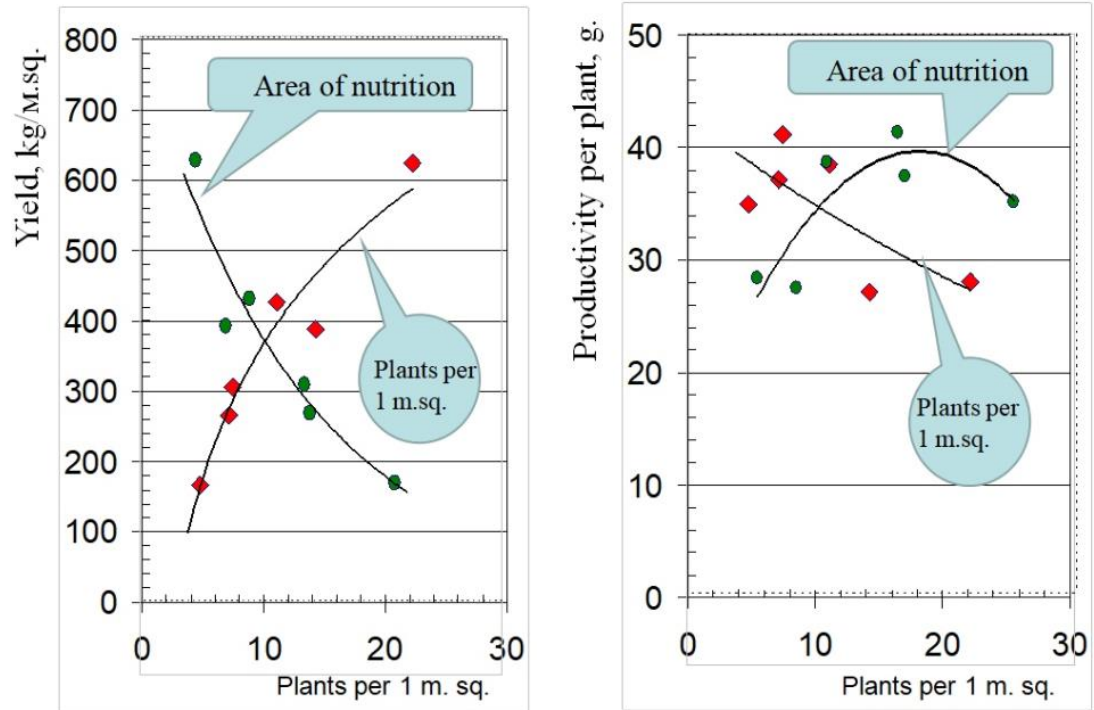

\section{B - root system}

Fig. 4. Graphic representation of the algorithm for creating agrocenosis of Echinacea purpurea for optimal productivity. 

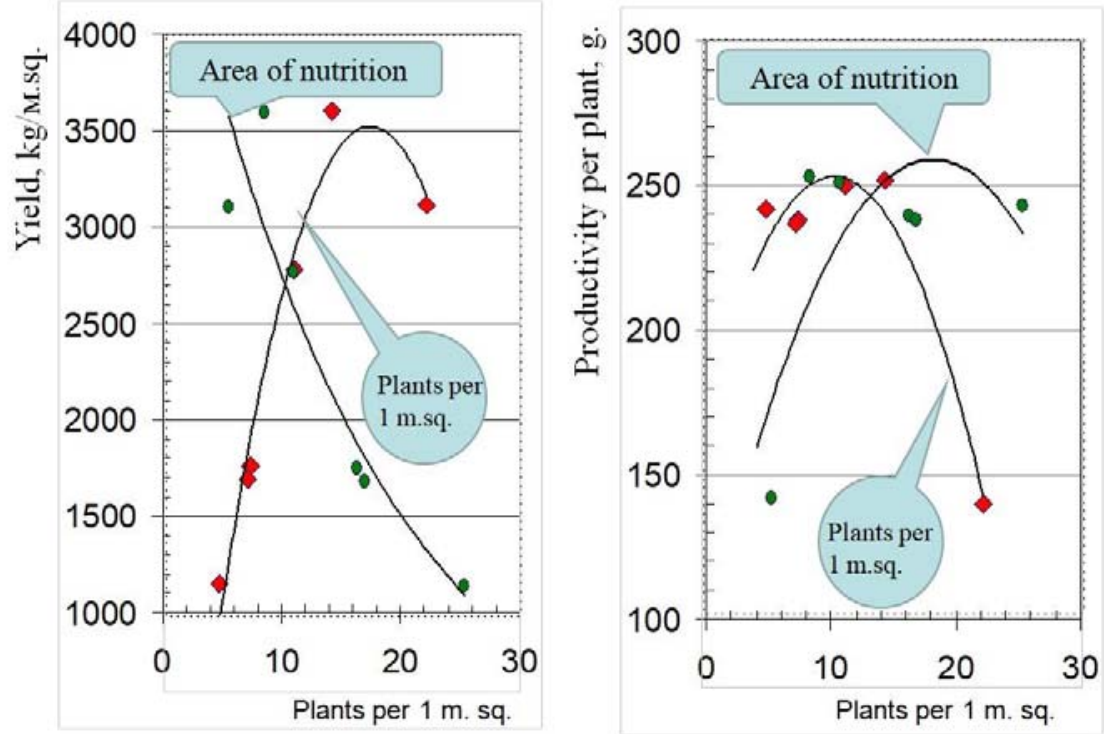

A - aboveground part
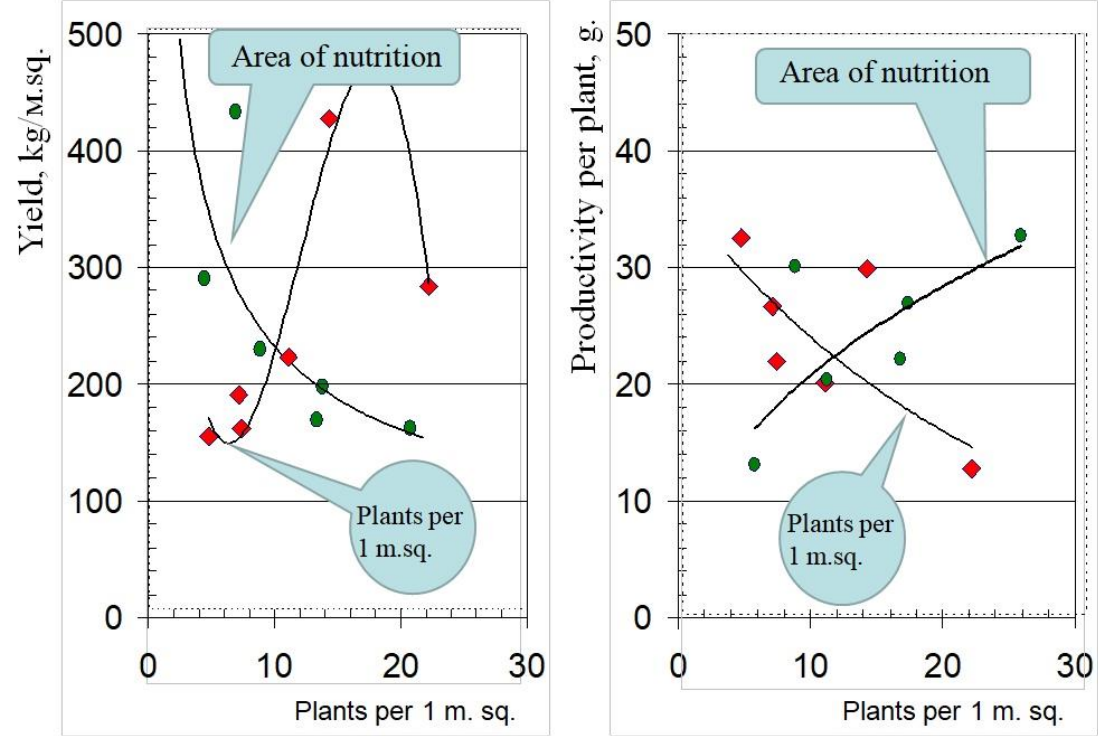

B - root system

Fig. 5. Graphic representation of the algorithm for creating agrocenosis of Echinacea pallida for optimal productivity.

One of the graphs shows the dependence of productivity and yield on the area of plant nutrition, the other - on the density of plants. After combining two trends, the coordinate systems of which are related to each other, we got two lines, the intersection of which indicates the optimal yield per square meter or the productivity of one plant and, 
accordingly, the number of plants per square meter. It should be noted that the algorithms presented below determine the optimal possible values. According to calculations, it is possible to program a larger yield by increasing the number of plants per unit area, but this will inevitably decrease the mass of individual plants.

Figure 4 shows a graphical representation of the algorithm for Echinacea purpurea. According to calculations, to obtain the optimal productivity (180-200 g per plant in our experiments) of the aboveground mass of Echinacea purpurea, it is necessary to form an agrocenosis with a density of 10 plants per $1 \mathrm{~m}^{2}$. When sown with $45 \mathrm{~cm}$ row spacing, this corresponds to 4.5 plants per linear meter. At the same time, an increase in yield can be achieved by compaction of the agrocenosis to a certain level. The optimal nutritional area for the root system is also 10 plants per $1 \mathrm{~m}^{2}$, but, the figure shows that an increase in the number of plants to $18-20$ plants $/ \mathrm{m}^{2}$ is the upper limit of the productivity of the root system and further compaction will not increase the yield of roots per unit area.

As can be seen in Figure 5, the productivity of the aboveground mass of Echinacea pallida of 240-250 $\mathrm{g}$ is optimal when creating a seeding density of $12-14 \mathrm{plants} / \mathrm{m}^{2}$, and this is practically the maximum possible level. Further compaction of the sowing causes a decrease in the yield of the aboveground mass. The optimum productivity of the root system is $34-36 \mathrm{~g}$ with a density of $12-14$ plants $/ \mathrm{m}^{2}$, and an increase in density also negatively affects its development. Our conclusions are close to the results of the studies [7] that show a high yield of raw materials with a high content of chicoric acid was achieved at an optimal density of Echinacea crops of 9-10 plants per $1 \mathrm{~m}^{2}$.

Thus, the yield of Echinacea purpurea can be regulated within certain limits by the density of the agrocenosis. At the same time, the optimal density can be considered to be $10-11$ plants $/ \mathrm{m}^{2}$, which corresponds to the schemes of 45 × $20-45$ × $25 \mathrm{~cm}$. For Echinacea pallida, the maximum yield is observed when creating agrocenosis with a density of 12-14 plants $/ \mathrm{m}^{2}$, which is close to the optimal placement. This corresponds to the schemes of $45 \times 13-45 \times 15 \mathrm{~cm}$. Planting plants with row spacings of $70 \mathrm{~cm}$ allows to get higher quality raw materials by reducing the part of the stems and increasing the part of the inflorescences. This feature must be taken into account when setting up industrial plantations of Echinacea pallida.

\section{Conclusions}

1. As a result of the studies carried out in 2006-2015, the main regularities of the development of plants of Echinacea purpurea and Echinacea pallida, depending on the placement schemes, were established. It was found that these types of echinacea reacted differently to spatial distribution in the cenosis due to biological characteristics. In thickened crops, Echinacea purpurea formed a limited number of generative shoots (3.34.0) and could increase them by 1.8-2.2 times with an increase in the distance between plants, while Echinacea pallida formed 2.7-5.7 stems regardless of the feeding area.

2. The obtained data were processed by the method of correlation analysis, which made it possible to construct correlation pleiades of echinacea productivity depending on the density of placement. It was found that for Echinacea purpurea, the mass of the aboveground part was mostly correlated to the mass of the stems $(r=0.879)$, the number of inflorescences $(r=0.735)$, and the mass of the root system $(r=0.815)$; the mass of the root system correlated with the mass of inflorescences, width and length of the leaf blade. For Echinacea pallida, the mass of the aboveground part was determined by the mass of the stems $(r=0.929)$, the mass of the inflorescences $(r=0.859)$ and the mass of the leaves $(r=$ 0.787); the mass of the root system did not have significant correlations with the studied parameters. 
3. The mathematical models were developed that allowed to determine the patterns of plant placement in the agrocenosis. According to calculations, the optimal productivity of Echinacea purpurea includes the aboveground mass of 180-200 g/plant and the weight of the root system of 20-25 g/plant; this is achieved by the density of the agrocenosis of 100110 thousand plants per hectare. For Echinacea pallida, the optimal productivity of the aboveground mass is $240-250 \mathrm{~g} / \mathrm{plant}$, and of rhizomes with roots $-34-36 \mathrm{~g} / \mathrm{plant}$; it can be achieved with an agrocenosis density of 120-140 thousand plants per hectare.

\section{References}

1. V.N. Samorodov, S.V. Pospelov, G.F. Moiseeva, \& A.V. Sereda, Pharmaceutical Chemistry Journal, 30(4), 245-251 (1996) https://doi:10.1007/BF02218771

2. R. Franke, R. Schenk, \& A. Nagell, Echinacea pallida (Nutt.) Nutt. - yield and echinacoside content (1999) https://doi:10.17660/ActaHortic.1999.502.25

3. S.V. Pospelov, \& V.N. Samorodov, The results of the study of representatives of the genus Echinacea Moench. in Poltava State Agrarian Academy, Medicinal Herbs: from past experience to new technologies. In honor of the 100th anniversary of the Echinacea research in Ukraine, Materials of the fourth International scientific and practical conference, $\quad$ Poltava, 63-79 (2015) https://www.pdaa.edu.ua/sites/default/files/node/2452/ivconfmedplant2015poltava.pdf

4. S.V. Pospelov, Phytochemistry, 3, 421-440 http://www.appleacademicpress.com/phytochemistry-volume-3-marine-sourcesindustrial-applications-and-recent-advances/9781771887618

5. Sandra Carol Miller, Echinacea: the genus Echinacea (CRC Press, 2004)

6. R. Romero, R.M. Barros Camba, A. Rigueiro \& J.L. Fernández Lorenzo, International Journal of Agricultural Science, 4(8), 232-240 (2014) https://doi.org/10.5281/ZENODO.2629813

7. N.W. Callan, T. Yokelson, S. Wall-MacLane, M.P. Westcott, J.B. Miller, \& G. Ponder, Journal of Herbs, Spices and Medicinal Plants, 11(3), 35-46 (2005) https://doi:10.1300/J044v11n03_04

8. K.M. Kleitz, M.M. Wall, C.L. Falk, C.A. Martin, M.D. Remmenga, \& S.J. Guldan, HortTechnology, 13(4), 631-636 (2003) https://doi:10.21273/horttech.13.4.0631

9. G.A. Parmenter, \& R.P. Littlejohn, New Zealand Journal of Crop and Horticultural Science, 25(2), 169-175 (1997) https://doi:10.1080/01140671.1997.9514003

10. A.S. Shalaby, S.E. El-Gengaihi, E.A. Agina, A.S. El-Khayat, \& S.F. Hendawy, Spices and Medicinal Plants, 5(1), 69-76(1997) https://doi:10.1300/J044v05n01_08

11. L.S. Tansi, S. Karaman, O. Toncer, \& S. Gedik, Turkish Journal of Field Crops, 20(2), 174-178 (2015) https: // doi: 10.17557 / tjfc.68776

12. Yi Lu, Ren-Shih Chung, Pi-Hui Suzi Chang, Effects of different nitrogen fertilizers and application rates on the growth and caffeic acid derivative contents of Echinacea purpurea (L.), 24-th International Symposium of the International Scientific Center of Fertilizers - Plant nutrition and fertilizer issues for specialty crops, Coimbra (Portugal), September 6-8, 23-24 (2016) https://doi.org/10.5073/BERJKI.2016.185.000 